\title{
Tibetan Medicine: An Effective Botanical Supplement for Peripheral Vascular Circulation
}

Vladimir Badmaev*

President, American Medical Holdings Inc, USA

\begin{abstract}
This review provides an instructive example of how a botanical and mineral formula known in the tradition of Tibetan medicine as Gabyr-Nirynga (camphor combination), denoted as formula No. 28, has evolved into a contemporary application through scientific research. This developmental pathway has led to a standardized nutraceutical with clinically proven efficacy in alleviation of a form of atherosclerosis-peripheral arterial disease (PAD). The systematic scientific research began in Switzerland in 1970s and resulted in five, double-blind clinical studies conducted in various European countries on cardiovascular use of the formula. The formula's mechanism of action has been described in Tibetan tradition based on its three groups of botanical and mineral ingredients: (1) main active ingredients, (2) auxiliary ingredients, and (3) components that offset the action of the first two groups and facilitate gastrointestinal absorption of the formula. Based on preclinical and clinical studies, several mechanisms of action relevant to alleviation of the PAD have been discussed, such as an increase in the threshold for platelet aggregation, lower total blood cholesterol and low-density lipoproteins (LDL), and prevention of blood lipid peroxidation. A study in an animal model of CNS inflammation, i.e. Experimental Allergic Encephalomyelitis (EAE), has shown that No. 28 can exert action as a biological response modifier improving response of the organism to chronic inflammation. This latter mechanism may be particularly relevant to the clinical benefits with No. 28 in patients with PAD, in view of cardiovascular disease increasingly considered as an outcome of a chronic inflammatory process. The integration of traditional knowledge of a botanical formula with science-derived pharmacology is referred in this paper as the Interactive Nutrients process.
\end{abstract}

Keywords: Camphor combination; Blood lipid peroxidation; Cardiovascular disease; PAD; PVD; cardiovascular; immune; Adaptrin; Badmaev 28; Padma 28

\section{Introduction}

The medicine of tomorrow may depend on a better understanding and integration of the traditional medical systems like Ayurveda or Tibetan Chinese traditions into contemporary medical practice. Although there has been lack of systematic written preservation of the traditional knowledge, continuous safe use of herbal and mineralbased treatments and time-accumulated wisdom that have withstood hundreds and sometimes thousands of years make these healing systems potentially suitable for preventive medicine and management of chronic degenerative conditions. The integration of traditional knowledge of a botanical formula with science-derived pharmacology is referred in this paper as the Interactive Nutrients process. The knowledge of botanical and mineral-based treatments may enrich an often one-sided, symptom-oriented, practice of contemporary pharmacology.

This review describes more than 30 years of preclinical and clinical evaluation of Tibetan botanical formula No. 28, primarily in the field of cardiovascular research. The outcome of this Interactive Nutrients process provided a novel strategy to enhance cardiovascular health.

\section{The Historical Background}

My personal interest in Tibetan medicine and India's Ayurveda comes from more than 170 years family history practicing those health care disciplines. My ancestors have combined western medicine, from the European schools, with Asian traditions [1-6]. Their ancestral knowledge of Tibetan medicine has come from the Asian part RussiaBuryatia, a land in the vicinity of Lake Baikal that had been influenced by a vast expansion of the Tibetan empire more than 1000 years ago [2,7]. The textbook of Tibetan medicine, $y$ Gyud-bzhi, or Four Roots, was originally written in Sanskrit in India during the 4th century, and its fate has been as complex as the history of Tibet and Tibetan medicine [8,9]. In the late 19th century, Four Roots was translated for the first time into a western language (Russian) by Drs. Sul-TimBadma (Alexander) and Zham-Saran-Badma (Peter) [8]. Their names were later changed by adding the Russian suffix "ev" to the family name of Badma or Padma, which in Sanskrit means "flower of lotus" to the names of Alexander and Peter Badmaev Sr [10].

My great granduncle, Dr. Peter Badmaev Sr., established the first Europe-based Tibetan pharmacy in St. Petersburg, Russia, in the late 1800s, and my Grandfather, Dr. Vladimir Badmaev Sr., established a medical practice and Tibetan pharmacy in the 1930s in Warsaw, Poland. They introduced a line of Tibetan-based botanical prescriptions, which have been further developed by other physicians in this family in Russia, Poland, Switzerland, and now, the United States [3-5].

This review summarizes the clinical research done on one particular prescription known in the Buryat language (transliteration) as "Gabyr Nirynga" and also known as Badmaev formula number 28. This formula has been recognized in the medical literature as Padma 28. The term Padma was chosen by a Swiss company established in 1965 jointly by Dr. Peter Badmaev Jr., my father, and Swiss businessman Karl Lutz; the company was dedicated to manufacturing the Tibetan botanical formulae based on Badmaev prescriptions.

*Corresponding author: Vladimir Badmaev, President, American Medical Holdings Inc, 1440-6 Forest Hill Rd, Staten Island, NY 10314, USA, Tel: (347) 4396237; E-mail: vebadmaev@attglobal.net

Received April 29, 2013; Accepted June 13, 2013; Published June 20, 2013

Citation: Badmaev V (2013) Tibetan Medicine: An Effective Botanical Supplement for Peripheral Vascular Circulation. J Cardiovasc Dis Diagn 1: 111. doi:10.4172/2329-9517.1000111

Copyright: (c) 2013 Badmaev V. This is an open-access article distributed under the terms of the Creative Commons Attribution License, which permits unrestricted use, distribution, and reproduction in any medium, provided the original author and source are credited. 
The number " 28 " does not refer to the number of ingredients in the formula, and the significance of number " 28 " and the numerical designations of other Tibetan formulae have yet to be determined. The formula No. 28 continuously developed by the author of this review paper is currently known in the market place as Adaptrin ${ }^{\circledR}$.

\section{Composition of Formula No. 28}

Formula No. 28 is composed of natural D (+) camphor, mineral calcium sulfate, and includes the following herbs: Lichen islandicus moss (Ach.) Iceland moss, Costus amarum root (C.B. Clarke) costus, spiral flag, Melia toosendan fruit (Linn.) neem, Elettaria cardamomum fruit (Malon.) cardamom, Pterocarpus santalinus wood (Linn.) red sandalwood, Terminalia chebulae fruit (Retz.) myrobalan, Amomum medicinalis fruit (L. Merrill) Jamaican pepper, Aegle sepiar fruit (L.Raffin) hardy orange, Aquilegia viridifolia leaves (Linn.) columbine, Plantago lanceolata herb (L.) ribwort, Glycyrrhiza glabra root (Linn.) licorice, Polygonum aviculare herb (Linn.) knotgrass, Potentilla aurea herb (L.) golden cinquefoil, Eugenia caryophyllata fruit (Spreng Wild.) cloves, Hedychium spicatum root (Ham ex. Smith) gingerlily, Sida cordifolia herb (Linn.) heart-leaved sida, Valeriana officinalis root (Linn.) valerian, Lactuca sativa leaves (Linn.) lettuce, Calendula officinalis flowers (Linn.) marigold.

Due to regulatory concerns, but also because historically the homeopathic monkshood in the No. 28 formula has not been essential, this ingredient is not present in the current formula. These components are blended according to standardized protocol into uncoated tablets with a distinctive taste, since traditionally the action of the formula starts with interaction with the taste buds.

The chemistry of the formula was studied by Samochowiec [11]. The entire formula was subject to sequential extraction and evaluation of the chemical constituents using high-pressure liquid chromatography (HPLC). The accompanying Table 1 lists known chemical constituents of No. 28.

\section{Clinical Trials in Peripheral Arterial Disease (PAD)}

When formula No. 28 was introduced in Switzerland in the 1960s by Dr. Peter Badmaev, Jr., its cardiovascular effects, known only from the physician's office practice, attracted the attention of clinical scientists, because of the lack of effective pharmacological therapies for vascular pathology. Dr.Werner Bubb, then vice president of the Swiss Medical Society, initiated efforts to conduct a controlled clinical trial of No. 28 in the treatment of Peripheral Arterial Disease (PAD). Bubb, who was familiar from his private practice with several of the Badmaev formulations, recruited Dr. Franz Hurlimann, a Zurich based angiologist.
Hurlimann was the first researcher to conduct a double-blind clinical trial with No. 28 [12]. He used the formula and the matching placebo in capsule form, which was different from the traditional uncoated tablets. The study involved 24 ambulatory patients (17 men and 7 women, $50-70$ years of age) with a history of PAD that was radiologically confirmed to be the pelvic/femoral artery occlusion type. The patients' PAD was clinically classified according to the Fontaine classification as stage II-B, that is, the patients' pain-free walking distance did not exceed $200 \mathrm{~m}$. Of the 24 patients, 12 reported nocturnal leg cramps and paresthesias. The patients were randomized: 13 patients were assigned to the active treatment group, and 11 were assigned to the placebo group. Each patient received three capsules twice daily for a period of 3 months.

The treated group showed a statistically significant $(p<0.02) 56 \%$ increase in the pain-free walking distance (measured on a treadmill) as compared with a $6 \%$ increase observed in the placebo group. Up to $70 \%$ of the 12 patients with nocturnal leg cramps and paresthesias before the treatment reported complete relief from the symptoms.

Hurlimann also conducted an open-field study with 10 patients ( 8 men and 2 women, 55-68 years old) with advanced PAD, or Fontaine classification stages III and IV of the disease. Those patients, in addition to having subjective feeling of pain during both walking and rest, also had trophic changes of the skin on the lower extremities. These openfield study patients received No. 28 in tablet form, three tablets twice daily, for a period of 3 months. Hurlimann reported beneficial results: pain measured at rest disappeared or was significantly reduced to tolerable levels in 7 of 10 patients, in addition to improved pain free walking distance. In both studies Hurlimann found no side effects of the treatment, and the formula was found compatible with other pharmacological therapy received by some of the patients, such as anticoagulant, antidiabetic, antihypertensive, antilipemic, and cardiac glycoside agents. Hurlimann concluded that the formula could be useful in combination with anticoagulant therapy for PAD [12].

Hurlimann's 1979 published results in Praxis, also known as Swiss Medical Review, stimulated much interest about formula No. 28 among the public and medical community [12]. After this publication, the Swiss Food and Drug Administration approved the formula as an over-the-counter supplement for the early stages of PAD. The official introduction of the botanical treatment for this serious medical condition polarized the Swiss medical community. Many physicians were convinced of the effectiveness of the formula because of the clinical evidence from Hurlimann's study as well as their own clinical experience. Other physicians remained skeptical, since Hurlimann's study was based on preliminary results. These critics also maintained that there was too little of the potential active principle(s) in the formula to exert the reported therapeutic effect.

\begin{tabular}{|l|l|}
\hline Phenolic acids and their glucosides & Salicylic acid, ferulic acid, syringin, vanillin, p-hydroxybenzoic acid, gentisic acid, p-hydroxyphenylbutyric acid, coumarin \\
\hline Glycosides & $\begin{array}{l}\text { Flavonoids (7-glycoside of luteolin, 7-glycoside of apigenin and quercetol); p-hydroxycyanic acid-glycosides (columbine); furan } \\
\text { glycosides (acubin) }\end{array}$ \\
\hline Aglycons & Isoquercitin, quercitin, luteolin, apigenin \\
\hline Polysaccharides & Inulin, starch, lichenin, dextrolichenin, vegetable mucilage \\
\hline Silicon compounds & Silicic acid, potassium silicate \\
\hline Organic acids & Isovaleric acid, citric acid, oxalic acid, formic acid. \\
\hline Volatile Oils & $\begin{array}{l}\text { Camphor; phenyl derivatives (eugenol, acetoeugenol, methyleugenol); terpenes (terpinene, terpineol); bicyclic terpenes (1-camphene, } \\
\text { 1-pinene, borneol); sesquiterpenes (limene, azullene); benzyl alcohol; heptyl alcohol; methylamyl-ketone }\end{array}$ \\
\hline Vegetable tannins & Catechinic acid, gellate acid \\
\hline Saponins & Glycyrrhizic acid \\
\hline Phenanthrene alkaloids & Aconite (not present in current formula) \\
\hline
\end{tabular}

Table 1: Chemical constituents of No. 28. 
Further evidence of the formula's efficacy came from a doubleblind study performed at the University of Bern, Switzerland, by Schrader et al. [13]. The study was conducted during a two-year period, 1982 to 1984. Schrader designed her study based on the previous experience of Hurlimann and concentrated on patients who had stage II PAD. The following inclusion criteria were selected: (1) clinical diagnosis of the disease, with at least 8-month duration; (2) maximal walking distance not exceeding $250 \mathrm{~m}$; (3) age of patients greater than 50 years; and (4) expected good compliance with the study's guidelines. Schrader's exclusion criteria included: (1) concurrent pharmacological treatment with vasoactive drug, (2) concurrent heart disease, and (3) wide variance in the walking distance.

The 43 patients selected for the study were randomly assigned to treatment and placebo groups. After a treatment-free interval of 14 days, 23 patients were treated for 4 months with the No. 28 , three capsules twice a day and 20 patients received matching placebo capsules.

After 4 months of treatment, patients who received the active formula increased the pain-free walking distance over baseline value on average by $66 \mathrm{~m}(100 \% ; p<0.002)$ as compared to the placebo group, who showed an average increase in walking distance over the baseline value of $30 \mathrm{~m}(46 \% ; p<0.01)$. The difference between the two groups was, however, not significant $(p=0.06)$. Maximal walking distance in the group receiving active treatment increased on average by $124 \mathrm{~m}$ over baseline value $(98 \% ; p<0.002)$ as compared with the statistically non-significant increase of walking distance in the placebo group of 27 $\mathrm{m}(21 \%)$.

Schrader also measured the degree of arterial occlusion from the pressure difference at rest between the systolic pressures of the upper arm and by the ultrasound Doppler method. The pressure difference at rest decreased significantly in the active treatment group, on average from $71 \mathrm{~mm} \mathrm{Hg}$ to $55 \mathrm{~mm} \mathrm{Hg}(p<0.03)$, as compared to a nonsignificant increase in the placebo group. None of the patients had to discontinue either active or placebo treatment due to side effects or poor tolerance. The reported side effects were randomly distributed among the both treatment groups, and were minor and transient in nature: four patients reported stomachaches, and three patients reported skin rash.

\section{Other European Studies}

As a result of the positive patient responses observed in both the Hurlimann [12] and Schrader et al. [13] studies, No. 28 became a popular herbal supplement used in several European countries by the patients with poor peripheral blood circulation. Subsequently more clinical studies were conducted in Europe, outside of Switzerland. In 1984, Samochowiec et al. conducted a double-blind study of No. 28 in PAD patients at the Department of Clinical Pharmacology of the Medical Academy in Szczecin, Poland [14]. The inclusion criteria for this study were maximal walking distance of less than $150 \mathrm{~m}$ and disease duration longer than 8 months. One hundred PAD patients were randomly assigned to the treatment groups: 19 women and 36 men received formula No. 28, and 18 women and 27 men received a placebo. The average age of study participants was 56 years in the active treatment group and 57 years in the placebo group. After a twoweek introductory period with no treatment, patients received active treatment or placebo in the form of capsules, two capsules twice daily for 4 months.

In the course of the 4-month treatment period, the average maximal walking distance for patients receiving No. 28 increased by $78 \mathrm{~m}$ over baseline value $(93 \% ; p<0.001)$, whereas no significant change occurred in the maximal walking distance of the placebo group. Biochemical testing revealed that active treatment resulted in significant reductions in the levels of cholesterol $(p<0.001)$, triglycerides $(p<0.01)$, and alphalipoproteins $(p<0.001)$ as compared to placebo. Active treatment also resulted in a significantly increased $(p<0.001)$ threshold for Adenosine Diphosphate (ADP)-dependent blood platelet aggregation, indicating improved blood viscosity and flow. All 100 patients completed the study, with no reports of side effects.

In 1993, Drabaek et al. from the Department of Clinical Physiology and Clinical Chemistry, Frederiksberg, Denmark, conducted another double-blind study of the formula with 36 PAD patients [15]. The Drabaek et al.'s study was done with 18 women and 18 men, with an average age over 60 years. The inclusion criteria for this study were a steady state of the disease for more than 6 months, maximal walking distance between 50 and $300 \mathrm{~m}$, and ratio between systolic blood pressures at the ankle and upper limb lower than 0.85 . Patients who suffered from chronic lung disease, diabetes mellitus, and diseases other than PAD that limited walking distance were excluded from the study group. The 36 patients were randomly assigned to receive either an active treatment in the form of capsules or matching placebo, two capsules twice daily for 4 months.

After 4 months of treatment, patients receiving No. 28 increased their pain-free walking distance on average from 52 to $86 \mathrm{~m}(65 \%$; $p<0.05)$, and the maximal walking distance from 115 to $227 \mathrm{~m}(97 \%$; $p<0.05)$. The placebo group did not show any significant changes in either their pain-free or maximal walking distance. Unlike Schrader, Drabaek could not demonstrate any significant changes in the difference between upper arm and ankle systolic pressure as a result of treatment with the herbal formulation. No side effects or dropout cases were reported in the study.

Another double-blind, randomized clinical trial to determine the efficacy of No. 28 in PAD patients was carried out by Smulski and Wojcicki from the Department of Internal Medicine, Szczecin District Hospital, Poland [16]. This study included 100 patients between the ages of 35 and 65 who had clinically diagnosed PAD. The subjects were randomly assigned to placebo and active treatment groups: 50 patients received active treatment, and 50 received a matching. Inclusion criteria were based on patient's history, positive physical examination results, maximum walking distance of less than $250 \mathrm{~m}$, and minimum disease duration of 6 months. Exclusion criteria were advanced PAD (Fontaine stages III and IV) and diseases other than PAD that affected walking ability. Patients received two capsules twice daily for 4 months.

The results of this study further confirmed the efficacy of No. 28 in treating PAD. Patients receiving the formula increased their maximum walking distance on average from 87.5 to $187.5 \mathrm{~m}$ (114\% increase; $p<0.001)$. Most of this increase occurred in the second half of the trial, particularly in the fourth month. Patients receiving the placebo showed a non-significant increase in walking distance, on average from 75 to $87.5 \mathrm{~m}$. Five placebo patients dropped out due to deterioration of their condition, and one patient receiving active treatment dropped out due to a newly diagnosed medical condition unrelated to PAD. Of the 49 patients receiving active treatment, 36 showed a clinically significant improvement, which was defined as more than a $50 \%$ increase in the maximum walking distance. In a subjective self-evaluation, 40 of the 49 patients receiving active treatment assessed the effectiveness of their treatment as good or very good, compared with 7 of 44 of the patients who received the placebo. The low-density lipoprotein (LDL) and total 
lipid levels decreased significantly in the group who received the herbal formula.

\section{Comparison of No. 28 with Synthetic Pharmaceuticals}

The summary of clinical studies with No. 28 performed on ethnically and culturally different study populations in Europe point to a significant clinical evidence of efficacy of the "condensed food", as No. 28 has been traditionally referred to, in subjects with poor peripheral circulation [12-16]. The efficacy data of No. 28 in PAD patients have been compared with synthetic pharmaceuticals tested in similar clinical trial designs [17-27]. As a parameter for the comparative evaluation, a percent of increase in maximal walking distance from various treatments has been chosen. Based on the comparison of the maximal walking distance, No. 28 emerges as an equal or superior therapeutic modality for treating PAD versus results with the following synthetic drugs: Pentoxifylline [Trental] Neftidrofuryl [Praxilene], Buflomedil [Loftyll], Bencyclan [Fludilat], Nifedipine [17-27].

\section{No. 28 in Cardiovascular Conditions besides PAD}

The double-blind randomized clinical studies confirmed effectiveness and safety of No. 28 in PAD led health care practitioners in Europe and the USA to use the formula in other cardiovascular conditions. The naturopathic MD, David Williams, well recognized for his practice and education on alternative treatments in the US has published a review on Adaptrin's cardiovascular and immune health promoting effects (Alternatives 1998 May, pp. 82-84). Intrigued by cardiovascular efficacy of No. 28, Dr. Panjwani, the former Director of Merck Research and Development, studied the formula in 34 elderly patients who suffered from gradually deteriorating mental acuity [28].

Each of the elderly patients in this study (median age, 59 years) had been seen in a physician's office for a number of years; all of them complained of worsening mental functions due to cardiovascular and cerebrovascular insufficiency. These patients were evaluated at the beginning of the study and then on a monthly basis. Six clinical indicators of cerebral function were used: (1) memory; (2) mental clarity and orientation; (3) overall energy and alertness; (4) general mental attitude, including depression; (5) sleep patterns, and (6) general subjective sense of well-being. Every day the patients took two tablets of No. 28 half an hour before breakfast and dinner. The study was conducted for 6 months, with a monthly evaluation of each participant. Among the 34 patients, 21 complied fully with the regimen throughout the 6 months of the study's duration.

The Panjwani et al. [28] study showed that the majority of patients treated with No. 28 benefited noticeably from taking the formula continuously for 6 months, as evaluated by self-rating of memory ( 21 of 21 improved), mental clarity and orientation (19 of 21 improved), overall energy and alertness ( 21 of 21 improved), sleep patterns (20 of 21 improved), general mental attitude (19 of 21 improved), and general well-being status ( 21 of 21 improved). None of the 21 patients who completed the study reported any side effects from the treatment or deterioration of the primary testing points. Of the 13 patients who did not complete the study, none reported side effects as the reason for earlier termination of the treatment. In conclusion, Panjwani concluded that No. 28 could potentially represent an important advance in gerontology, in safe and effective care for the aging population.

In summary, the outcome of the outline of the Interactive Nutrient process with No. 28 points to a potential food-derived cardiovascular support, with a benefit of a long standing track record of safety.

\section{Mechanism of Action of No. 28 in Preclinical and Clinical Studies}

The preclinical and clinical evidence suggest that the mechanism of No. 28 in cardiovascular health is multifactorial, i.e. improving blood rheology, the immune response to the endothelial injury and to the arterial plaque, and by alleviating chronic inflammation associated with cardiovascular disease. In the 1970s and concurrently with Hurlimann's study, a platelet aggregation study was performed with No. 28 in in vivo conditions, based on the formula's hypothesized antithrombotic activity, (Badmaev V, Personal communication Laboratory of Experimental Surgery, Davos, Switzerland) [12,29]. The formula was fed to healthy rabbits ( 1 to 1.5 tablets daily) for 7 days, showed the collagen-induced inhibition of platelet aggregation. The effect of No. 28 on platelet aggregation was confirmed in clinical studies [14]. During the 16 weeks' PAD study, the threshold for ADP -dependent platelet aggregation increased by $100 \%(p<0.001)$.

The additional rheological mechanism of No. 28 may come from improved blood lipid composition. No. 28 when administered to rats fed with a high-fat diet (HFD) for 12 weeks, reduced blood levels of total cholesterol and triglycerides, and increased alpha-lipoproteins as compared to the untreated animals. ${ }^{31}$ The levels of lipid endoperoxides, as measured by malonedialdehyde or MDA (metabolite of oxidized plasma lipids) levels were reduced more than two-fold in No. 28 receiving animals. MDA formation correlates with lipid peroxidation, systemic inflammation, arterial plaque formation and increased synthesis of thromboxane A2, a prostaglandin with vasoconstricting and blood clotting promoting properties. The role of No. 28 in lipid peroxide formation prevention was confirmed in cardiovascular patients in studies conducted by Samochowiec et al. [30,31].

The multifactorial mechanism of No. 28 has also been shown in the in vitro and in vivo experiments measuring the immune response parameters $[32,33]$. The immunological studies were initiated due to historical use of No. 28 in chronic inflammatory conditions (e.g. hepatitis), autoimmune conditions (e.g. neurodegenerative and demyelinating conditions) and allergic conditions (e.g. bronchial asthma). The in vitro study showed that water extract from the entire formula selectively improved T-lymphocyte immunological performance in the lymphocytes obtained from peripheral blood of patients with lowered immune response (volunteers with clinically diagnosed multiple sclerosis), evaluated in T-cell rosette and mitogen stimulation tests [32].

The in vivo evaluation of No. 28 immunological properties was done in a model of systemic inflammatory condition, the Experimental Allergic Encephalomyelitis (EAE) model in SJL mice [33,34]. This chronic inflammatory condition affects predominantly the central nervous system (EAE is regarded as an experimental model for studying the multiple sclerosis pathology) prominently involving blood vessels. The water extract of No. 28 administered in drinking water to mice resulted in a significantly lower mortality rate in animals with EAE and improved neurological symptoms as compared to untreated controls.

In summary, the cardiovascular mechanism of No. 28 may be a result of improved blood homeostasis and normalized immunological dis-regulation, which may quell chronic inflammation and collectively improve the CVD and PAD conditions.

\section{Discussion and Conclusions}

Peripheral Arterial Disease (PAD) is a manifestation of 
atherosclerosis in the arteries of the lower extremities. PAD is a common disorder in the general population, with prevalence increasing with age and vascular risk factors, e.g. diet, lifestyle and diabetes. The condition can be asymptomatic or with feeling of pins and needles, or extremity "falling asleep" but the most classic symptomatic expression is intermittent claudication, i.e. pain in lower extremities upon exertion. Those affected with PAD have increased mortality rates, mainly due to cardiovascular and cerebrovascular expressions of atherosclerotic disease. Endovascular surgical repair offers the benefit, however recurrence of arterial occlusion is often. Pharmacological treatment depends on blood-thinners and comes at cost of serious side effects. Therefore safe, time proven and clinical research supported nutritional help offered by No. 28 formula is a welcome development.

In accordance with the three essential elements (triadic) theory in Tibetan and Ayurvedic medicines, No. 28 contains three categories of herbal and mineral ingredients that consist of main active ingredients supplemental ingredients, and ingredients that prevent any undesirable effects arising from the pharmacological action of the first two groups of ingredients and facilitate gastrointestinal absorption of the formula [1-3]. This concept in Tibetan herbal pharmacology has been developed with the perception of safe and effective treatment in form of "condensed food" or the multicomponent botanical formulae. According to this tradition, only the combined mechanism of action can be credited with a broad, safe, and effective pharmacological activity of the formula.

One of the paradoxes for No. 28 regimen, often brought up by skeptics, is that none of the individual ingredients of the formula has been used in a clinically meaningful pharmacological dose. In view of the documented clinical efficacy, it becomes apparent that a mechanism other than the dose-dependent action of individual ingredients operates with the formula. A plausible explanation could be that sub-therapeutic doses of the individual ingredients are summarily able to activate the body's natural defenses against the disease. This mechanism correlates with Tibetan medicine providing the body's own disease-fighting abilities are more helpful than the techniques devised by man against a disease.

The collective clinical experience of physicians in my family has used the formula to treat conditions with different manifestation and underlying pathology, e.g. diseases due to immunosuppression as well as others due to overt stimulation of the immune system [4]. The therapeutic effect of No. 28 demonstrates that the formula is acting as an adaptogen or, better defined as a bioprotectant-that is, a substance that protects homeostasis by the action of prevention and intervention against the specific pathology, e.g. caused by free radicals [34].

The No. 28 formula should be regarded as a classic paradigm of a safe and effective formula refined by the Interactive Nutrient process of preclinical and clinical studies of the traditional botanical formula. The dose of No. 28 used in the cited clinical studies has been up to 2 times higher than would be recommended by a Tibetan practitioner. The clinical researchers not convinced with efficacy of sub-pharmacological doses of No. 28 resorted to higher daily doses administered continuously throughout duration of the study for several weeks. That regimen precluded gradual dosing of No. 28 typically recommended in Tibetan tradition and derived from understanding that the majority of diseases are:

1. Not caused by an abrupt insult but result from long-standing abuse of the organism;
2. The gastrointestinal system is central in the pathogenesis of most diseases;

3. Any disease affects all the organs and body systems, despite the obvious manifestation associated with the body organ/ system affected most;

4. The role of any treatment is to support but not to replace the organism's natural defense mechanism against disease.

According to this understanding, most treatments should be aimed at a correspondingly gradual recovery from a disease. To insure that improvement related to therapy is gradual, the treatment with an herbal formula usually starts with one third of the maximum therapeutic dose over a period of weeks. Based on the reaction of the individual patient, the dose may be increased, and after accomplishing the therapeutic goal gradually tapered off. Usually therapy spans from one to four months and is followed by a one-month break and re-evaluation. If improvement has not been accomplished the cycle of treatment is repeated. The one-month break between cycles was designed not only for re-evaluation of the condition but also to enhance the organism's own physiological defense mechanisms against the disease.

Traditionally No. 28 formula is recommended 1 tablet 3 times daily between meals, although patients with body weight over $200 \mathrm{lb}$. may benefit from 2 tablets 3 times daily. For best results, the dose should be built up gradually starting with 1 tablet daily week one, 2 tablets daily week two, then, 3 tablets daily from week three on. After 4 months at maximum dose and the one-month break, 1 tablet daily should be taken as a maintenance dose. Traditionally, the formula is pressed in form of uncoated tablet to convey organoleptic qualities of herbs. Based on the Oriental principle," food is your medicine," it is believed that the starting point of biological action is in contact with oral mucosa and the taste buds. The clinical studies due to its double-blinded nature had to resort to use of identical looking capsules also masking the organoleptic properties of the No. 28 formula versus placebo.

This Tibetan understanding of gradual approach to managing health and disease with low dose escalating to a higher dose of the supplemental herbs may be gaining understanding in view of the above discussed bioprotectant mechanism of a compound that protects homeostasis by the action of prevention and intervention against the specific pathology [34]. The bioprotectant mechanism may be critical to the management of chronic inflammation. It is inflammation as a lingering, rather than an acute, process that is increasingly attracting attention and requires different approaches than inhibition of the inflammatory pathways. Healing of the chronic inflammatory process rather than at all costs inhibiting the inflammation, may lead to a proper therapeutic approach in many poorly understood and treated conditions. Cardiovascular disease is no longer considered solely due to dislipidemia, but rather a disease process characterized by low-grade inflammation of the vascular lining and an inappropriate "wound healing response" of the blood vessels. The No. 28 formula emerges as a hopeful nutritional approach to cardiovascular health and management of cardiovascular disease.

\section{References}

1. Berthenson LB (1906) Uber russische Buddistah und die sogenannte tibetanische medzin. St. Petersburgh. Med Wochenschr 24: 248-257.

2. Semicov BV (1935) Die tibetische medizin bei den Burjaten. Janus 39: 1-36.

3. Kowalewski K (1973) Wladimir Badmajeff; Tibetan doctor in Europe. J Res Indian Med 8: 101-109. 
Citation: Badmaev V (2013) Tibetan Medicine: An Effective Botanical Supplement for Peripheral Vascular Circulation. J Cardiovasc Dis Diagn 1: 111. doi:10.4172/2329-9517.1000111

4. Badmajew P Jr, Badmajew V Jr, Park L (1982) Healing Herbs, Berkeley, Calif. Red Lotus Press 90.

5. Vladimir Badmaev (1983) Father-son MD team have Tibetan legacy. Med Tribune (USA) 13:13

6. Ross M (1990) Tibet: Ancient remedies gain new acceptance as research explores 'mysterious' medicines. Medical Post (Canada) 10: 14.

7. Sarton G (1927) The time of Jabir Ibn Haiyan. In: Haiyan JI. Introduction to the History of Science. Baltimore: Williams \& Wilkins. 520-542.

8. Kunzang J (1973) Tibetan Medicine. Wellcome Institute of the History of Medicine. 8-28.

9. Winder M (1973) Tibetan Medicine. In: Kunzang J (ed.). Wellcome Institute of the History of Medicine. 1-7

10. Badmaev V, Majeed M Ayurveda medicine of positive health. Energy Times 47-51.

11. Samochowiec L (1983) Theoretische und chemisch-pharmacodinamische untersuchunger von Padma 28 (lecture). 1st International Convention on Tibetan Medicine. Venice, Italy

12. Hürlimann $F$ (1978) A lamaistic formula for the treatment of peripheral arterial occlusive diseases (author's transl). Schweiz Rundsch Med Prax 67: 14071409.

13. Schrader R, Nachbur B, Mahler F (1985) Die Wirkung des tibetanischen krauterpraparates Padma 28 auf die claudicatio intermittens. Schweiz Med Wochenschr 115: 752-756.

14. Samochowiec L, Wojcicki H, Kosmider K, Dadej R, Smulski H (1987) Wirksamkeitsprufung von Padma 28 bei der behandlung von patienten mit chronischen arteriellen durchblut- ungsstorungen Herba Polonica 33: 29.

15. Drabaek H, Mehlsen J, Himmelstrup H, Winther K (1993) A botanical compound Padma 28, increases walking distance in stable intermittent claudication. Angiology 44: 863-867.

16. Smulski HS, Wojcicki J (1995) Placebo-controlled, double-blind trial to determine the efficacy of the Tibetan plant preparation Padma 28 for intermittent claudication. Alt Ther Health Med 1: 44-49.

17. Porter JM, Bauer GM (1982) Pharmacologic treatment of intermitten claudication. Surgery $92: 966-971$

18. Völker D (1978) Treatment of arterial diseases with trental 400. Results of a double-blind study. Med Welt 29: 1244-1247.

19. Porter JM, Cutler BS, Lee BY, Reich T, Reichle FA, et al. (1982) Pentoxifylline efficacy in the treatment of intermittent claudication: multicenter controlled double-blind trial with objective assessment of chronic occlusive arterial disease patients. Am Heart J 104: 66-72.

20. Accetto B (1982) Beneficial hemorheologic therapy of chronic peripheral arterial disorders with pentoxifylline: results of double-blind study versus vasodilatornylidrin. Am Heart J 103: 864-869.

21. Pohle W, Hirche H, Barmeyer J, Schümichen C, Hoffmann G (1979) Doubleblind study of naftidrofuryl-hydrogen oxalate in peripheral arterial occlusive disease. Med Welt 30: 269-272.

22. Maass U, Cachovan M, Alexander K (1983) Effect of interval training on walking distance, hemodynamics and ventilation in patients with intermitten claudication. II. Changes of hemodynamics and ventilation. Vasa 12: 326-332.

23. Trübestein G, Balzer K, Bisler H, Klüken N, Mahfoud Y, et al. (1982) Buflomedil in arterial occlusive disease. Results of a controlled study. Dtsch Med Wochenschr 107: 1957-1961.

24. Holle W, Schneider B (1982) Bencyclan, pentoxifylline und placebo be peripheren durchblutungsstoerungen. Dtsch Med Wschr 107: 1957-1981.

25. Lindgärde $F$, Jelnes $R$, Björkman $H$, Adielsson G, Kjellström T, et al. (1989) Conservative drug treatment in patients with moderately severe chronic occlusive peripheral arterial disease. Scandinavian Study Group. Circulation 80: 1549-1556.

26. Chacón-Quevedo A Eguaras MG, Calleja F Garcia MA, Roman M, et al. (1994) Comparative evaluation of pentoxifylline, buflomedil, and nifedipine in the treatment of intermittent claudication of the lower limbs. Angiology 45: 647653.

27. Badmaev V (1999) Tibetan Medicine. In: Essentials of Complementary and Alternative Medicine by Wayne B. Jonas, Jeffrey S. Levin (eds.); published by Lippincott Williams \& Wilkins.

28. Panjwani HK, Priestley J, Lewis AE (1987) Clinical evaluation of Padma 28 in treatment of senility and other geriatric circulatory disorders: A pilot study. Alt Med 2: 11-17.

29. Platelet aggregation. Davos Report. 1974. [Personal communication Badmaev V]

30. Samochowiec L, Wojcicki J (1987) Effect of Padma 28 on lipid endoperoxides formation. Herba Polonica 33: 219-222.

31. Samochowiec L, Janiec W, Wojcicki J (1985) Influence of Padma 28 on the oxidation of palmitic acid-1-14C to 14CO2 . Polbiopharm Rep 21: 41-49.

32. Badmajew V, Brzosko WJ, Dabrowski MP, Dabrowska-Berstein BK (1982) Padma 28: An immunoregulatory substance in vitro. Int J Immunopharmaco 4: 382

33. Badmaev V, Kozlowski PB, Schuller-Levis GB, Wisniewski HM (1999) The therapeutic effect of an herbal formula Badmaev 28 (padma 28) on experimental allergic encephalomyelitis (EAE) in SJL/J mice. Phytother Res 13: 218-221.

34. Badmaev V, Majed M (1995) Adaptogens and bioprotectants. Nutrition Science News. 